\title{
MATHEMATICAL NOTES
}

Review of Elementary Mathematics and Science

\author{
PUBiLISHED BY
}

\author{
THE EDINBURGH MATHEMATICAL SOCIETY \\ EDITED BY WILLIAM ARTHUR, M.A.
}

No. $28 \quad$ October 1930

\section{An Extension of Clifford's Circle Chain.}

\section{By John Dovgall.}

In elementary plane geometry there is a very well known theorem attributed to Wallace (1806) which runs:

Theorem I. If four lines are given in a plane, the circumscribing circles of the triangles which they form three by three are concurrent.

Call the point of concurrence the Wallace point of the four lines. If now five lines are taken, we get five Wallace points by leaving out each line in turn. Miquel's Theorem is that these five Wallace points lie on a circle, say the Miquel circle of the five lines. It was shown by Clifford (1870) that these results can be extended indefinitely. Thus from six lines we get six Miquel circles by leaving out each line in turn: these six circles pass through a point, say the Clifford point of the six lines. Then from seven lines we get seven Clifford points: these lie on a circle, say the Clifford circle of the seven lines. Next, the eight Clifford circles of eight lines pass through a point; at the next step nine such points lie on a circle; and so on similarly as long as we please.

The question suggests itself : what are the corresponding theorems on a sphere, when instead of straight lines we take great circles, or more generally, circles whose planes are concurrent? To answer this question is the object of the present note. The theorems given are not really new, ${ }^{1}$ though possibly they may be a little difficult to recognize in the forms in which they are now stated.

In the first place, by a simple inversion from a centre either in the given plane or outside it, we can transform the straight lines

\footnotetext{
1 See in particular Professor Baker's beautiful book cited below.
} 
from which we started into circles through a point. This generalization is trivial, but it suggests another which is more far-reaching. To obtain it, note that Wallace's theorem, when transferred to a sphere, may be stated in the form:

Theorem II. If $A, B, C, A^{\prime}, B^{\prime}, C^{\prime}$ are six points on a sphere, and if the circles $A B C, A B^{\prime} C^{\prime}, A^{\prime} B C^{\prime}, A^{\prime} B^{\prime} C$ are concurrent, so also are the circles $A^{\prime} B^{\prime} C^{\prime}, A^{\prime} B C, A B^{\prime} C, A B C^{\prime}$.

A little consideration suggests that the sphere has little to do with it, and that the following statement, in terms of points and planes, may be correct:

Theorem III. If $A, B, C, A^{\prime}, B^{\prime}, C^{\prime}$ are six points in space, and if the planes $A B C, A B^{\prime} C^{\prime}, A^{\prime} B C^{\prime}, A^{\prime} B^{\prime} C$, meet in a point $(D)$, then the planes $A^{\prime} B^{\prime} C^{\prime}, A^{\prime} B C, A B^{\prime} C, A B C^{\prime}$ also meet in a point $\left(D^{\prime}\right)$.

This is actually a true theorem, first published by Moebius (1828). It is closely allied to the theorem of Pappus (Pascal's theorem for a conic which has degenerated into two straight lines), and to the projective theory of involution of points on a line.

Theorem III may be deduced ${ }^{1}$ in a moment from the well known result that the sides and diagonals of a complete quadrangle are cut in involution by any transversal.

Otherwise, we may use the theorem that a quadric surface through seven of the eight common points of three quadrics goes through the eighth also. Thus the eight points $A, \ldots, D^{\prime}$ lie by the data on the quadric formed by the two planes $A B^{\prime} C^{\prime} D, A^{\prime} B C D^{\prime}$; similarly with $A^{\prime} B C^{\prime} D, A B^{\prime} C D^{\prime}$; and with $A^{\prime} B^{\prime} C D, A B C^{\prime} D^{\prime}$. Therefore the quadric consisting of the two planes $A B C D, A^{\prime} B^{\prime} C^{\prime}$, which passes through seven of the points, passes through $D^{\prime}$, so that $D^{\prime}$ lies in the plane $A^{\prime} B^{\prime} C^{\prime \prime}$.

Note that the method of the latter proof shows that if the seven points other than $D^{\prime}$ lie on a quadric, then $D^{\prime}$ also lies on the quadric; which in effect is Wallace's Theorem.

The two tetrads of points $A, B, C, D^{\prime}$ and $A^{\prime}, B^{\prime}, C^{\prime}, D$ are called Moebius tetrads by Professor Baker. Veblen and Young call them Moebius tetrahedra. The figure has the property that the plane through any three points of either tetrad passes through a point of the other tetrad.

${ }^{1}$ H. F. Baker, Principles of Geometry, Vol. I, p. 61.

2 Veblen and Young, Projectire Geometry, Vol. I, pp. 105, 326. 
Having got our theorem in terms of planes and points, we may now start over again with six points $A, B, C, A^{\prime}, B^{\prime}, C^{\prime}$ lying on a sphere, and such that the planes $A B C, A B^{\prime} C^{\prime}, A^{\prime} B C^{\prime}, A^{\prime} B^{\prime} C$ meet in a point, but we suppose now that the point does not lie on the sphere. Then, to get back to circles, we take the sections of the sphere by the various planes. We therefore have:

Theorem IV. If $A, B, C, A^{\prime}, B^{\prime}, C^{\prime}$ are six points on a sphere, and if the planes of the circles $A B C, A B^{\prime} C^{\prime}, A^{\prime} B C^{\prime}, A^{\prime} B^{\prime} C$ meet in a point $(D)$, then the planes of the circles $A^{\prime} B^{\prime} C^{\prime}, A^{\prime} B C, A B^{\prime} C, A B C^{\prime}$ also meet in a point $\left(D^{\prime}\right)$.

Now, when the plane of a circle on a sphere passes through a fixed point, the circle is orthogonal to a fixed (real or imaginary) circle, viz. to the section of the sphere by the polar plane of the fixed point. In fact circles on a sphere are orthogonal when their planes are conjugate with respect to the sphere.

Our generalization (IV) of Wallace's Theorem may therefore be stated in a form which applies to circles on a sphere and in a plane alike:

Theorem $V$. If $A, B, C, A^{\prime}, B^{\prime}, C^{\prime}$ are six points in a plane or on a sphere, and if the circles $A B C, A B^{\prime} C^{\prime}, A^{\prime} B C^{\prime}, A^{\prime} B^{\prime} C$ are orthogonal to a circle, then the circles $A^{\prime} B^{\prime} C^{\prime}, A^{\prime} B C, A B^{\prime} C, A B C^{\prime}$ are also orthogonal to a circle.

More generally still, we may take all eight points $A, B, \ldots$ of the two Moebius tetrads to be outside the sphere, and state Theorem III in terms of the sections $a, b, \ldots$ of the sphere by the polar planes of the points :

Theorem VI. If $a, b, c, a^{\prime}, b^{\prime}, c^{\prime}$ are six circles on a sphere or in a plane, and if the four orthogonal circles of the respective triads abc, $a b^{\prime} c^{\prime}, a^{\prime} b c^{\prime}, a^{\prime} b^{\prime} c$ are orthogonal to a circle, then the four orthogonal circles of the respective triads $a^{\prime} b^{\prime} c^{\prime}, a^{\prime} b c, a b^{\prime} c, a b c^{\prime}$ are also orthogonal to a circle.

This may also be stated in the form:

Theorem VII. If $a, b, c, d, a^{\prime}, b^{\prime}, c^{\prime}, d^{\prime}$ are eight circles on a sphere or in a plane, and if each of the seven tetrads of circles

$\begin{array}{ll}a b^{\prime} c^{\prime} d & a^{\prime} b c d^{\prime} \\ a^{\prime} b c^{\prime} d & a b^{\prime} c d^{\prime} \\ a^{\prime} b^{\prime} c d & a b c^{\prime} d^{\prime} \\ a b c d & \end{array}$

is orthogonal to a circle, then the tetrad $a^{\prime} b^{\prime} c^{\prime} d^{\prime}$ is also orthogonal to. a circle, or, in other words, the circles $a^{\prime} b^{\prime} c^{\prime} d^{\prime}$ are coorthogonal. 
We can now prove the extension of the Clifford chain. The method of proof is practically identical with that given by Coolidge ${ }^{1}$ for the Clifford theorem, and by Baker ${ }^{2}$ for the corresponding theorem in terms of planes and points. If we wish to avoid imaginaries, we may do so by replacing "circle" throughout by "plane," and "orthogonal" by "conjugate," i.e. "conjugate with respect to the fixed sphere."

We start from a basic circle $\Omega$ on the sphere. Take any number of circles all orthogonal to $\Omega$; denote these by single symbols $1,2,3, \ldots$ Take next any circles orthogonal to pairs of these; denote the chosen circle orthogonal to 1 and 2 by 12 ; and so on. Then there is one circle orthogonal to the three of this last set whose names involve three symbols only; we denote the circle orthogonal to 12, 13, 23 by 123; and so on. Next we have four circles of this new set involving only four symbols, e.g. 234, 134, 124, 123 involving the symbols $1,2,3,4$. In Theorem VII take 1 for $a$, 234 for $a^{\prime}$, etc., as in the scheme:

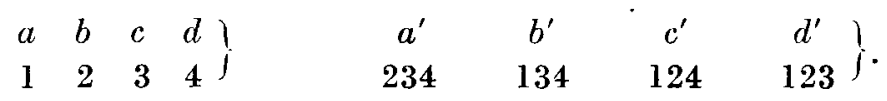

Then we have $a, b, c, d$ all orthogonal to $\Omega ; a, b^{\prime}, c^{\prime}, d$ to 14 ; and so on, as in the scheme:

\begin{tabular}{|c|c|c|c|}
\hline$a b^{\prime} c^{\prime} d$ & 14 & $a^{\prime} b c d^{\prime}$ & 23 \\
\hline$a^{\prime} b \quad c^{\prime} d$ & 24 & $a b^{\prime} c d^{\prime}$ & 31 \\
\hline $\begin{array}{llll}a^{\prime} & b^{\prime} & c & d \\
a & b & c & d\end{array}$ & $\begin{array}{c}34 \\
\Omega\end{array}$ & $a b c^{\prime} d^{\prime}$ & 12 \\
\hline
\end{tabular}

it being noted that two circles are orthogonal, by the above definitions, if their names differ by one symbol only.

Hence, by Theorem VII, $a^{\prime}, b^{\prime}, c^{\prime}, d^{\prime}$, i.e. the four circles 234, $134,124,123$ are orthogonal to a circle, which we call 1234 .

Next, we take five circles $1,2,3,4,5$; and we show that the five circles derived from these as above, viz.

$$
2345, \quad 1345, \quad 1245, \quad 1235, \quad 1234,
$$

are coorthogonal. We prove this by showing that any four of them are coorthogonal; say the four involving the symbol 5 . The proof is exactly that just given, except that we replace $\Omega$ by 5 , and adjoin the symbol 5 to every one of the combinations of symbols used

1 J. L. Coolidge, A Treatise on the Circle and Sphere, p. 90.

2 H. F. Baker, l. c. vol. IV, pp. 18, 29. 
above; i.e. for $a, b_{s} c, d$ we take $15,25,35,45$, and for $a^{\prime}, b^{\prime}, c^{\prime}, d^{\prime}$ we take $2345,1345,1245,1235$; so that $a, b^{\prime}, c,^{\prime} d$ are now orthogonal to 145 , and so on. We have therefore a circle orthogonal to 2345,1345 , $1245,1235,1234$; this we denote by 12345 .

At the next step we adjoin a new symbol 6 , in addition to the 5 already adjoined, and use the same proof as before. The chain can in this way be extended without limit:

Theorem VIII. An unending chain of circles can be defined, such that each circle can be named by a finite number of different symbols, and such that any two circles are orthogonal to each other if the name of one can be got from that of the other by simply cutting out or adding one symbol. The chain starts from a single circle, whose symbol for the purposes of this statement is to be considered a blank; and there is a circle in the cinain corresponding to every combination of different symbols.

\section{Alternative Forms of Expression for Hermite's Determinant.}

\section{By Sir Thomas Muir.}

(1) Apparently it was in 1854 that Hermite first drew attention to the special determinant which now bears his name. It may be defined as being such that every two of its elements that are conjugate in position are conjugate-complex in form: and as a consequence its matrix is the sum of two matrices one of which is axisymmetric and the other zero-axial skew.

(2) Although Hermite had clear evidence that the determinant was imaginary only in appearance, he does not seem to have made any effort to obtain an expression for it free of $\sqrt{-1}$. Such an expression is first met with, almost casually, in a paper of Clebsch's of 1859 , in which he has occasion to consider the latent roots of a Hermitant. His result we may formulate for ourselves thus: Any Hermitant of the third order is expressible as the difference of a determinant and a ternary quadric, the former being the determinant of the axisymmetric portion of the matrix and the latter having for its discriminant the same determinant: for example:-

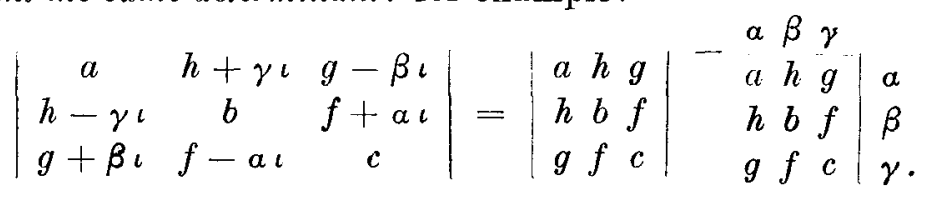

